\title{
Myocardial viability and cardiac dyssynchrony as strong predictors of perioperative mortality in high-risk patients with ischemic cardiomyopathy having coronary artery bypass surgery
}

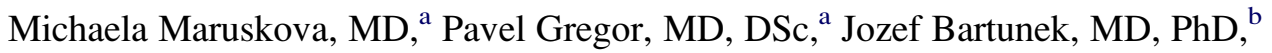 \\ Jaroslav Tintera, $\mathrm{PhD},{ }^{\mathrm{c}}$ and Martin Penicka, $\mathrm{MD}, \mathrm{PhD}^{\mathrm{a}}$
}

\begin{abstract}
Objective: Myocardial viability and left ventricular dyssynchrony are important predictors of long-term outcomes in patients with ischemic left ventricular dysfunction. The objective of this study was to test the hypothesis that assessment of myocardial viability and left ventricular dyssynchrony will predict perioperative mortality in high-risk patients with ischemic left ventricular dysfunction having coronary artery bypass surgery.
\end{abstract}

\begin{abstract}
Methods: The study consisted of 79 consecutive patients with ischemic cardiomyopathy (age $65 \pm 9$ years; $81 \%$ men; ejection fraction $30 \% \pm 6 \%$ ) and logistic European system for cardiac operative risk evaluation $>10 \%$ having coronary artery bypass surgery. Myocardial viability was assessed by delayed contrast-enhanced magnetic resonance imaging. Left ventricular dyssynchrony was calculated using tissue Doppler from measurements of regional electromechanical coupling times in left ventricular basal segments before coronary artery bypass surgery.
\end{abstract}

Results: Twenty $(25.3 \%)$ patients died within 30 days following coronary artery bypass surgery. Survivors $(\mathrm{n}=59)$ showed a larger extent of viable myocardium $(6.9 \pm 3.6$ viable segments vs $3.4 \pm 3.3$ viable segments, $P<.001)$ and smaller left ventricular dyssynchrony $(75 \pm 5 \mathrm{~ms}$ vs $179 \pm 83 \mathrm{~ms}, P<.001)$ than nonsurvivors. The presence of significant dyssynchrony ( $\geq 105 \mathrm{~ms}$ ) and absence of myocardial viability ( $<5$ viable segments) independently predicted 30-day mortality with hazard ratio $3.26,95 \%$ confidence interval 1.61 to $8.33(P<.01)$ and hazard ratio $1.72,95 \%$ confidence interval 1.59 to $1.89(P<.01)$, respectively. All but 2 patients $(94.1 \%)$ with viable myocardium and without left ventricular dyssynchrony survived coronary artery bypass surgery as compared with only $12(52.2 \%)$ patients with nonviable myocardium and severe dyssynchrony $(P<.001)$.

Conclusions: In high-risk patients with ischemic left ventricular dysfunction having coronary artery bypass surgery, both myocardial viability and left ventricular dyssynchrony are important predictors of perioperative outcome. Assessment of myocardial viability and left ventricular dyssynchrony should be a routine part of the preoperative evaluation of these patients.

Myocardial revascularization using coronary artery bypass surgery (CABG) is associated with improved long-term survival in some patients with coronary artery disease and severe left ventricular (LV) dysfunction compared with conservative management. ${ }^{1,2}$ However, in these patients, the potential long-term benefits of revascularization must be weighed against the high periprocedural risks. ${ }^{1-3}$ The

From the Cardiocenter, Department of Cardiology, ${ }^{a} 3$ rd Faculty of Medicine, Charles University and the University Hospital Kralovske Vinohrady, Prague, Czech Republic; Cardiovascular Center, ${ }^{\mathrm{b}}$ OLV Hospital, Aalst, Belgium; and Department of Radiology, ${ }^{\mathrm{c}}$ Institute of Clinical and Experimental Medicine, Prague, Czech Republic.

This work was supported by Research Grant IGA 8524 and by the Charles University, Prague, Research Project MSM 0021620817, which was awarded by the Czech Ministry of Education.

Received for publication March 27, 2008; revisions received Sept 26, 2008; accepted for publication Nov 20, 2008.

Address for reprints: Martin Penicka, MD, PhD, Cardiocenter, Department of Cardiology, 3rd Faculty of Medicine, Charles University and the University Hospital Kralovske Vinohrady, Srobarova 50, 10034 Prague, Czech Republic (E-mail: penicka@fnkv.cz).

J Thorac Cardiovasc Surg 2009;138:62-8

$0022-5223 / \$ 36.00$

Copyright (c) 2009 by The American Association for Thoracic Surgery doi:10.1016/j.jtcvs.2008.11.040
European system for cardiac operative risk evaluation (EuroSCORE) is a widely accepted scoring system used to predict immediate postoperative outcomes after CABG. $^{4,5}$ High EuroSCORE levels have been associated with high perioperative mortality. However, in patients with severe LV dysfunction, additional factors not integrated in the EuroSCORE system, such as the extent of viable myocardium or cardiac dyssynchrony, may be important determinants of perioperative outcomes. ${ }^{1-3,6}$ Several studies ${ }^{1,2,6}$ have demonstrated a strong relationship between the amount of myocardial viability and survival following CABG surgery in patients with ischemic LV dysfunction. In one of our recent studies, ${ }^{3}$ the presence of severe LV dyssynchrony prior to CABG was significantly associated with elevated 30-day mortality in patients having moderate systolic heart failure. This suggests that the predictive accuracy of the EuroSCORE may be lower in patients with ischemic LV dysfunction compared with patients with normal systolic function. The reduced predictive value of the EuroSCORE in patients with LV dysfunction may complicate clinical decision making, particularly in critically ill patients at high perioperative risk. 


$$
\begin{aligned}
& \text { Abbreviations and Acronyms } \\
& \mathrm{CABG}=\text { coronary artery bypass surgery } \\
& \text { CI }=\text { confidence interval } \\
& \text { EuroSCORE }=\text { European system for cardiac } \\
& \text { operative risk evaluation } \\
& \mathrm{HR} \quad=\text { hazard ratio } \\
& \mathrm{LV} \quad=\text { left ventricular } \\
& \text { NYHA }=\text { New York Heart Association } \\
& \text { ROC }=\text { receiver operating characteristics } \\
& \text { STS }=\text { Society of Thoracic Surgeons } \\
& \text { mortality risk score }
\end{aligned}
$$

Therefore, the objective of the present study was to test the hypothesis that assessment of myocardial viability and LV dyssynchrony will predict perioperative mortality and will improve the predictive accuracy of the EuroSCORE in high-risk patients with ischemic LV dysfunction having CABG.

\section{METHODS}

\section{Patients}

Between January 1, 2005 and December 31, 2005, 1252 patients had CABG at our institutions. The prospective study consisted of $79(6.3 \%)$ consecutive patients (age $65 \pm 9$ years; $81 \%$ men) having isolated CABG who met the following criteria: (1) stable LV dysfunction (LV ejection fraction $<40 \%$, for at least 3 months; to be eligible for the study, the presence of qualifying LV dysfunction had to be already documented at least 3 months before enrollment); (2) standard ( $\geq 3$ months) heart failure medication including angiotensin-converting enzyme/AT1 inhibitors and B-blockers if tolerated; (3) logistic EuroSCORE $>10 \%$. Patients with acute coronary syndrome or hospitalization for worsening heart failure in the previous 3 months; any valvular heart disease requiring surgery, pacemakers, or internal defibrillators; and contraindications for magnetic resonance were excluded from the study. All patients recruited for the study had CABG surgery as the sole procedure. None of the patients had concomitant LV remodeling, aneurysmectomy, mitral valve repair, or the MAZE procedure. The study was approved by the ethical committee. All patients gave a written informed consent prior to participation.

\section{Protocol}

In the week leading up to CABG surgery, each patient had assessment of myocardial viability through contrast enhanced magnetic resonance imaging; LV dyssynchrony was evaluated using pulsed-wave tissue Doppler. Perioperative risk was calculated using the logistic EuroSCORE. Patients were then followed for 30 days postsurgery to assess 30-day mortality.

\section{Assessment of Myocardial Viability by Magnetic Resonance Imaging}

Delayed contrast-enhanced electrocardiogram-gated magnetic resonance images (1.5-T scanner Siemens Symphony, Erlangen, Germany) were acquired using a phased-array receiver coil during breath-holds. ${ }^{7}$ Short-axis images were acquired every $5 \mathrm{~mm}$ from base to apex. Imaging was done 20 minutes after intravenous injection of a gadolinium-based contrast agent (for dose of $0.2 \mathrm{mmol} / \mathrm{kg}$ ). Images were analyzed off-line by an independent operator. The extent of hyperenhanced tissue was assessed in each segment (16-segment model) of the left ventricle. Segments with $\leq 50 \%$ of transmural hyperenhancement were considered viable. ${ }^{6}$

\section{Assessment of LV Dyssynchrony by Tissue Doppler Echocardiography}

Echocardiograms were performed using a standard commercial ultrasound system equipped with tissue Doppler imaging (Vivid 7, VingmedGeneral Electric, Horten, Norway). Tissue Doppler was performed in pulsed-wave mode. In brief, in 3 apical (4-, 3-, and 2-chamber) views, longitudinal myocardial velocities were recorded in 6 basal segments of the left ventricle. ${ }^{3}$ Gain and filters were adjusted to obtain an optimal tissue signal. Myocardial velocities were recorded at end expiration at a sweep speed of $100 \mathrm{~mm} / \mathrm{s}$. All recordings were stored both in digital (raw data) format and on S-VHS videotape for off-line analysis. The mean from 3 consecutive beats was taken for each measurement. Echocardiographers were blinded to clinical follow-up data.

To assess the extent of LV dyssynchrony, the time delay between the onset of QRS complex on the surface electrocardiogram and the onset of the systolic velocity waves on the tissue Doppler imaging recordings were assessed in each basal LV segment. ${ }^{8}$ Dyssynchrony was calculated as the difference between the shortest and the longest time delay in the 6 basal segments. Hence, LV dyssynchrony represents a delay in the onset of contraction between the segment with the earliest and the segments with the latest systolic wall motion. Intra- and interobserver variability for the assessment of LV dyssynchrony was $7.1 \%$ and $8.2 \%$, respectively.

\section{EuroSCORE}

EuroSCORE is a risk stratification system for the prediction of early mortality in patients having cardiac surgery. ${ }^{5}$ It has been in use since 1999 . The EuroSCORE has a total of 17 factors, consisting of 9 patient-related factors, 4 factors reflecting the preoperative cardiac status, and 4 factors that depend on the timing and nature of the surgical procedure. Together, the scores predict an approximate percentage for mortality.

\section{Statistical Analysis}

Data are presented as mean \pm standard deviation. Two-sided unpaired Student $t$ test was used as appropriate. The Fisher exact test was used to compare categorical variables. The receiver-operating characteristics (ROC) curves were constructed to assess optimal cutoff values for the number of dysfunctional viable segments and LV dyssynchrony to predict the 30-day mortality. Independent predictors of the 30-day mortality were identified using the Cox proportional hazard model and expressed as a hazard ratio (HR) and 95\% confidence interval (CI). All analyses were conducted using SPSS software (version 13).

\section{RESULTS \\ Baseline Characteristics}

Twenty $(25.3 \%)$ patients died within 30 days following CABG (in-hospital deaths). All patients died of pump failure leading to low-output syndrome, multiorgan failure, or sepsis. No patient died of arrhythmias. Table 1 shows baseline characteristics in survivors and nonsurvivors. All patients had left main $(19 \%)$ or 3 -vessel disease $(81 \%)$. The majority $(86 \%)$ of included patients showed symptoms of congestive heart failure and $47.8 \%$ had severe heart failure (New York Heart Association [NYHA] class III-IV). Angina pectoris was an indication for CABG in $72 \%$ of patients, and isolated heart failure symptoms in $28 \%$ of patients. Prior to $\mathrm{CABG}$, survivors had milder heart failure symptoms and higher $\mathrm{LV}$ ejection fractions (both $P<.05$ ) and tended to have lower systolic pulmonary artery pressure $(P=.051)$ than patients who died. Also, survivors had significantly 
TABLE 1. Baseline characteristics of the 30-day survivors and nonsurvivors

\begin{tabular}{|c|c|c|c|}
\hline & Survivors $(\mathbf{n}=\mathbf{5 9})$ & Nonsurvivors $(\mathbf{n}=\mathbf{2 0})$ & $P$ value (survivors vs nonsurvivors) \\
\hline Age (y) & $65 \pm 9$ & $66 \pm 10$ & NS \\
\hline Male sex, n (\%) & $47(80)$ & $17(85)$ & NS \\
\hline Diabetes mellitus, n (\%) & $19(28)$ & $7(35)$ & NS \\
\hline Hypertension, $\mathrm{n}(\%)$ & $45(76)$ & $15(75)$ & NS \\
\hline Obesity, n (\%) & $14(24)$ & $6(30)$ & NS \\
\hline Prior myocardial infarction, $\mathrm{n}(\%)$ & $44(74)$ & $12(60)$ & NS \\
\hline Chronic obstructive pulmonary disease, $\mathrm{n}(\%)$ & $15(25)$ & $4(20)$ & NS \\
\hline Prior stroke, n (\%) & $6(10)$ & $3(15)$ & NS \\
\hline Peripheral vascular disease, n (\%) & $16(27)$ & $5(25)$ & NS \\
\hline Serum creatinine $>200 \mu \mathrm{mol} / \mathrm{L}, \mathrm{n}(\%)$ & $6(10)$ & $4(20)$ & NS \\
\hline Angina pectoris, $\mathrm{n}(\%)$ & $44(75)$ & $13(65)$ & NS \\
\hline NYHA functional class III-IV, n (\%) & $23(34)$ & $14(70)$ & .021 \\
\hline Congestive heart failure, $\mathrm{n}(\%)$ & $50(85)$ & $18(90)$ & NS \\
\hline \multicolumn{4}{|l|}{ Preoperative medication } \\
\hline Loop diuretics & $52(88)$ & $19(95)$ & NS \\
\hline ACE/AT1 inhibitors & $47(80)$ & $15(75)$ & NS \\
\hline ß-blockers & $44(75)$ & $14(70)$ & NS \\
\hline Digoxin & $16(27)$ & $6(30)$ & NS \\
\hline Left main disease, $\mathrm{n}(\%)$ & $11(19)$ & $4(20)$ & NS \\
\hline Three-vessel disease, $\mathrm{n}(\%)$ & $48(81)$ & $16(80)$ & NS \\
\hline LV ejection fraction $(\%)$ & $30 \pm 6$ & $27 \pm 5$ & .042 \\
\hline Systolic pulmonary artery pressure (mm $\mathrm{Hg}$ ) & $41 \pm 8$ & $45 \pm 10$ & .051 \\
\hline Dysfunctional viable segments (n) & $6.9 \pm 3.6$ & $3.4 \pm 3.3$ & $<.001$ \\
\hline Pre-CABG LV dyssynchrony (m)s & $75 \pm 55$ & $179 \pm 83$ & $<.001$ \\
\hline Logistic EuroSCORE (\%) & $14.5 \pm 4.6$ & $14.3 \pm 3.8$ & NS \\
\hline
\end{tabular}

$A C E$, Angiotensin-converting enzyme; $C A B G$, coronary artery bypass surgery; EuroSCORE, European system for cardiac operative risk evaluation; $L V$, left ventricular; $N Y H A$, New York Heart Association.

more viable myocardium and less LV dyssynchrony (both $P<$.001) than nonsurvivors. In contrast, the EuroSCORE and other baseline clinical characteristics were similar in both groups.

\section{Operative Data and 30-Day Outcomes}

Operative data and 30-day outcomes are detailed in Table 2. All patients had on-pump CABG. Mean bypass and ischemic times were similar in both groups. Complete revascularization was achieved in the majority ( $84 \%$ ) of patients. Post-CABG, nonsurvivors had a higher prevalence of low-output syndrome and renal failure requiring dialysis. In contrast, prevalence of perioperative myocardial infarction, stroke, bleeding, and sepsis was similar.

\section{Prediction of 30-Day Mortality}

The ROC curves were constructed to analyze the accuracy of baseline variables in predicting 30-day mortality (Figure 1). Preoperative LV dyssynchrony, with a cutoff value of $\geq 105$ $\mathrm{ms}(\mathrm{n}=23)$, showed the highest accuracy to predict clinical events. Fourteen $(61 \%)$ of 23 patients with severe dyssynchrony $(\geq 105 \mathrm{~ms})$ died compared with $6(11 \%)$ of the 56 patients without dyssynchrony. The sensitivity and specificity of this criterion to identify nonsurvivors were $70 \%$ and $85 \%$, respectively. The number of dysfunctional but viable segments, with a cutoff value $<5$ segments, had lower accu- racy (area under the curve 0.71), and the EuroSCORE failed to differentiate between survivors and nonsurvivors (area under the curve 0.47). In the Cox regression analysis, LV dyssynchrony $\geq 105 \mathrm{~ms}$ and $<5$ dysfunctional but viable segments were identified as independent predictors of 30-day mortality with HR $3.26,95 \%$ CI 1.61 to $8.33(P<.01)$ and HR $1.72,95 \%$ CI 1.59 to $1.89(P<.01)$, respectively.

Table 3 shows the EuroSCORE-derived predicted mortality versus real 30-day mortality in patients divided according to the presence or absence of myocardial viability and LV dyssynchrony. In the study group as a whole, 30-day mortality was significantly higher than predicted by the EuroSCORE $(P=.02)$. In patients with significant myocardial viability, EuroSCORE predictions and the real mortality were similar. In contrast, EuroSCORE significantly underestimated the perioperative risk in patients without viable myocardium, in whom the 30-day mortality was 3 times higher than EuroSCORE predictions $(P=.02)$. In the subset of patients with a large extent of myocardial viability and without LV dyssynchrony, EuroSCORE severely overestimated the risk, with real mortality being $59 \%$ lower than EuroSCORE predictions. All but 2 patients $(94.1 \%)$ with viable myocardium and without LV dyssynchrony survived CABG surgery, compared with only $12(52.2 \%)$ patients with nonviable myocardium and severe dyssynchrony $(P<.001)$. 
TABLE 2. Operative data and the 30-day outcomes

\begin{tabular}{|c|c|c|c|}
\hline & Survivors $(\mathbf{n}=\mathbf{5 9})$ & Nonsurvivors $(\mathbf{n}=\mathbf{2 0})$ & $P$ value (survivors vs nonsurvivors \\
\hline Preoperative intra-aortic balloon pump, n (\%) & 0 & 0 & NS \\
\hline On-pump CABG surgery, n (\%) & $59(100)$ & $20(100)$ & NS \\
\hline Cardiopulmonary bypass time (min) & $122 \pm 48$ & $126 \pm 50$ & NS \\
\hline Ischemic time (min) & $90 \pm 40$ & $95 \pm 46$ & NS \\
\hline Number of grafts & $3.3 \pm 0.9$ & $3.5 \pm 1.0$ & NS \\
\hline Complete revascularization, $\mathrm{n}(\%)$ & $50(85)$ & $16(80)$ & NS \\
\hline Low-output syndrome, n (\%) & $19(32)$ & $20(100)$ & $<.001$ \\
\hline Postoperative intra-aortic balloon pump, $\mathrm{n}(\%)$ & $17(29)$ & $18(90)$ & $<.001$ \\
\hline Perioperative myocardial infarction, $\mathrm{n}(\%)$ & $2(3)$ & $1(5)$ & NS \\
\hline Perioperative stroke, $\mathrm{n}(\%)$ & $2(3)$ & $1(5)$ & NS \\
\hline Bleeding requiring reoperation, $\mathrm{n}(\%)$ & $3(5)$ & $1(5)$ & NS \\
\hline Sepsis, $\mathrm{n}(\%)$ & $2(3)$ & $3(15)$ & NS \\
\hline Renal failure requiring dialysis, $\mathrm{n}(\%)$ & $6(10)$ & $7(35)$ & .016 \\
\hline
\end{tabular}

$C A B G$, Coronary artery bypass surgery.

\section{DISCUSSION}

The present study enrolled high-risk patients with ischemic LV dysfunction having CABG. In these patients, who had excessive perioperative mortality risks, the decision regarding the optimal therapeutic strategy is often very difficult. Our study found that the EuroSCORE alone was unable to discriminate 30-day survivors from nonsurvivors. Patients who died had lower LV ejection fraction, higher NYHA class, less myocardial viability, and more LV dyssynchrony compared with survivors. Myocardial viability and LV dyssynchrony turned out to be the strongest predictors of perioperative outcome. The absence of viable myocardium tripled the risk of perioperative death compared with EuroSCORE-predicted mortality. The presence of viable myocardium with concomitant absence of LV dyssynchrony was associated with reduced mortality compared with EuroSCORE estimates. Hence, in high-risk patients with ischemic LV dysfunction, the choice of optimal therapeutic strategies should be based both on the EuroSCORE and on the assessment of myocardial viability and LV dyssynchrony.

\section{Prediction of Perioperative Outcome in Patients with Ischemic LV Dysfunction}

EuroSCORE has been validated as a system to predict immediate postoperative outcome after adult cardiac surgery. ${ }^{5}$ Both the additive and logistic EuroSCORE have been proven accurate in identifying individuals at high risk of the perioperative death in the general population of patients having cardiac surgery. ${ }^{4,5}$ However, in the present study, the accuracy of the EuroSCORE alone to identify 30-day non survivors was low. This suggests that although reliable with regard to the general population, EuroSCORE may be less reliable in specific subsets of patients, such as those with LV dysfunction. Dysfunctional LV myocardium consists of variously scarred myocardium, hibernating and stunned muscle, and dyssynchrony. Although all these parameters were shown to be important predictors of perioperative and long-term outcome, they are not incorporated into the EuroSCORE system. ${ }^{1-3,6,9,10}$ In the present study, the presence of viable myocardium stabilized perioperative mortality at the level predicted by EuroSCORE. In contrast,

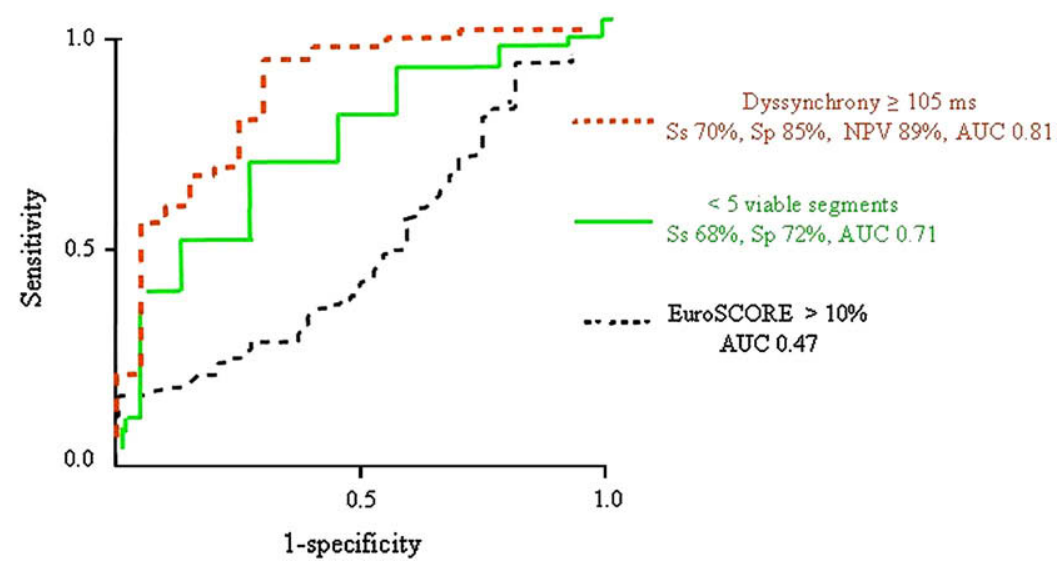

FIGURE 1. The receiver-operating characteristic curves to predict the 30-day mortality for left ventricular dyssynchrony, myocardial viability, and the EuroSCORE. AUC, Area under curve; EuroSCORE, European system for cardiac operative risk evaluation; $N P V$, negative predictive value; $S p$, specificity; Ss, sensitivity. 
TABLE 3. EuroSCORE-derived predicted mortality versus real 30-day mortality according to the myocardial viability and LV dyssynchrony

\begin{tabular}{|c|c|c|c|c|}
\hline & All patients $(n=79)$ & VIAB $(n=48)$ & NonVIAB $(\mathbf{n}=\mathbf{3 1})$ & VIAB noDYS $(n=34)$ \\
\hline EuroSCORE-predicted mortality $(\%)$ & 14.4 & 14.5 & 14.3 & 14.1 \\
\hline Real 30-d mortality ( $\%)$ & $25.3^{*}$ & 14.6 & $41.9^{*}$ & 5.9 \\
\hline $\begin{array}{l}\text { Percentage } 30 \text {-d mortality risk change by } \\
\text { assessment of VIAB and/or DYS prior to } \\
\text { CABG }(\%)\end{array}$ & NA & No change & $\uparrow 293$ & $\downarrow 59$ \\
\hline
\end{tabular}

the absence of viability was associated with excessive mortality risk, which was severely underestimated by EuroSCORE. Of note, the pre-CABG assessment of myocardial viability was not used for patient selection. In our study, patient selection for CABG surgery was guided by symptoms and the extent of coronary atherosclerosis. All patients with angina were considered to be for eligible for surgery. CABG surgery has been shown to improve survival in patients with angina compared with conservative therapy. ${ }^{11}$ In case of isolated heart failure symptoms, the decision to perform CABG surgery was guided by the extent of coronary artery disease. All patients with left main or proximal 3-vessel disease were considered eligible for myocardial revascularization regardless of presenting symptoms or viability status. Of note, in the Coronary Artery Surgery Study registries, the patients with left main or proximal 3-vessel disease were shown to gain the greatest survival benefit from myocardial revascularization compared with medical therapy. ${ }^{11}$ The role of viability studies in the management of patients with ischemic LV dysfunction having CABG remains unclear. Many observational studies demonstrated low benefit to performing revascularization in patients without viable myocardium. ${ }^{1,2}$ However, in the majority of these studies, the viability status was not used for the selection of patients for the CABG surgery. In contrast, several studies ${ }^{12,13}$ showed similar outcomes after CABG regardless of viability status, and also patients with nonviable myocardium had lower long-term mortality compared with medical therapy. Thus, in the absence of any randomized data and conflicting results from observational studies, it is difficult to decline revascularization in patients with left main or proximal 3-vessel disease based only on the absence of viable myocardium. Yet the data from the present study suggest that in the high-risk patients with heart failure and nonviable myocardium, an alternative approach to surgical revascularization should be strongly considered, such as palliative percutaneous coronary intervention or medical therapy.

Cardiac dyssynchrony complicates the course of systolic heart failure. Onset of dyssynchrony is associated with a deterioration of LV function and worsens the prognosis. ${ }^{9,10}$ We have previously demonstrated a strong relationship between the extent of LV dyssynchrony and 30-day outcome in patients with moderated heart failure having $\mathrm{CABG}^{3}$ The present study extends these findings to high-risk indi- viduals with ischemic LV dysfunction. We found that the absence of dyssynchrony halved the mortality predicted by EuroSCORE in patients with viable myocardium. In other words, the presence of significant dyssynchrony eliminated the advantage associated with increased viable myocardial mass. Patients with the most severe dyssynchrony had the worst outcomes.

The mechanism underlying the adverse effects of LV dyssynchrony on immediate postoperative outcome might be explained as follows: the prevalence of significant LV dyssynchrony in the cohort of patients with ischemic LV dysfunction referred for $\mathrm{CABG}$ ranges from $36 \%$ to $51 \%$. $^{3}$ The reduction of ischemia by CABG is associated with resynchronization of contraction in only $29 \%$ of patients with a high degree of preoperative dyssynchrony. ${ }^{3}$ Nevertheless, these patients show prompt recovery of contractile function after switching off the cardiopulmonary bypass and have uncomplicated postoperative courses. In contrast, patients with persistent dyssynchrony $(71 \%)$ do poorly following termination of cardiopulmonary bypass. We observed that some segments were not contracting because they were nonviable, and the viable segments started contracting but in an uncoordinated (dyssynchronous) fashion. The contraction of some of these viable segments were so delayed that it occurred during diastole, which meant contraction was taking place while the aortic valve was closed. Therefore, it was not surprising that these patients frequently had persistent low-output syndrome and died from cardiac or multiorgan failure within a few days. Dyssynchrony correction, with biventricular pacing, has been shown to improve contractile function and survival in patients with advanced systolic heart failure. ${ }^{14,15}$ Of note, in our study, presence of severe heart failure was associated with an increased 30-day mortality. One may speculate that in patients with significant LV dysfunction and LV dyssynchrony, implantation of a biventricular pacemaker prior to CABG or temporal biventricular pacing immediately after surgery could improve postoperative outcomes.

\section{Limitations}

In the present study, the perioperative mortality rate was higher compared with other studies in patients with LV dysfunction. ${ }^{16-22}$ The high mortality rate observed in the present study may be related to the selection criteria 
applied. First, only high-risk patients with end-stage coronary artery disease (left main [LM] 19\%; three-vessel disease $[3-\mathrm{VD}]=81 \%$ ) and EuroSCORE $>10 \%$ were enrolled in the study. Second, the majority $(86 \%)$ of included patients had congestive heart failure symptoms and $47.8 \%$ had severe heart failure (NYHA class IIIIV). Of note, nonsurvivors had higher prevalence of NYHA class III to IV heart failure than survivors. The significant proportion of enrolled patients with severe heart failure could be explained by using high EuroSCORE as the main selection criterion. Several authors showed that the pre-CABG presence of congestive heart failure is one of the main independent predictor of operative mortality. ${ }^{17-20}$ Of note, these studies with lower 30-day mortality enrolled smaller proportion of patients with heart failure compared with the present study. ${ }^{16-22}$ Finally, $39 \%$ of patients had nonviable myocardium. In a high-risk patient with low ejection fraction and significant comorbidities, assessment of myocardial viability is sometimes used as a basis for clinical decision making as to whether or not to perform CABG surgery. Patients with nonviable myocardium are likely to be declined by cardiosurgeon. In the present study, such selection was not performed, and hence the prevalence of patients with nonviable myocardium may be higher than in the previous reports. Corroborating this hypothesis, in our series, the excessive mortality was observed predominantly in patients with nonviable myocardium. In contrast, the remaining patients showed mortality in the range of $10 \%$ to $15 \%$, which corresponds with the preoperative expectations in high-risk patients with significant LV dysfunction.

In the present study, the perioperative risk was assessed using the EuroSCORE system, which is a standard system used both in the Czech Republic and within Europe. However, there are also other risk prediction models such as the Society of Thoracic Surgeons mortality risk score (STS), ${ }^{23}$ which is often used by North American surgeons. Despite some differences between the EuroSCORE and the STS model, both risk scores are very similar in predictive performance, at least in patients having isolated CABG surgery. Previous work demonstrated that the EuroSCORE was very reliable in assessing operative risk in the North American population. ${ }^{24}$ Moreover, several studies showed superior accuracy of the EuroSCORE to predict perioperative mortality compared with the STS score..$^{25,26}$ Nashef and colleagues ${ }^{24}$ found that the EuroSCORE-predicted mortality was remarkably similar to the observed mortality in a sample of over one-half million patients having CABG or valve surgery. They recommended the use of the EuroSCORE over the STS score. Another advantage of the EuroSCORE may rest on ease of its use. The STS model, with more than 50 clinical variables, is a very comprehensive tool to implement at the bedside. In contrast, the EuroSCORE is very easy to use at the bedside. This suggests that despite its simplicity, the EuroSCORE provides comparably accurate assessment of perioperative risk as the more complex STS score.

In conclusion, the findings of the present study indicate that in high-risk patients with ischemic LV dysfunction having $\mathrm{CABG}$, both myocardial viability and LV dyssynchrony are important predictors of perioperative outcome. A combination of EuroSCORE, viability, and dyssynchrony testing allows for superior risk stratification prior to surgical revascularization as compared with the EuroSCORE alone. For this reason, assessment of myocardial viability and LV dyssynchrony should become a routine part of the preoperative evaluation of these patients.

We are indebted to Martina Havlikova for her excellent administrative support throughout the study.

\section{References}

1. Chareonthaitawee P, Gersh BJ, Araoz PA, Gibbons RJ. Revascularization in severe left ventricular dysfunction: the role of viability testing. J Am Coll Cardiol. 2005;46:567-74.

2. Allman KC, Shaw LJ, Hachamovitch R, Udelson JE. Myocardial viability testing and impact of revascularization on prognosis in patients with coronary artery disease and left ventricular dysfunction: a meta-analysis. J Am Coll Cardiol. 2002;39:1151-8.

3. Penicka M, Bartunek J, Lang O, Medilek K, Tousek P, Vanderheyden M, et al. Severe left ventricular dyssynchrony is associated with poor prognosis in patients with moderate systolic heart failure undergoing coronary artery bypass grafting. J Am Coll Cardiol. 2007;50:1315-23.

4. Biancari F, Kangsniemi OP, Luukkonen J, Vuorisalo S, Satta J, Pokela R, et al. EuroSCORE predicts immediate and late outcome after coronary artery bypass surgery. Ann Thorac Surg. 2006;82:57-61.

5. Nashef S, Rogues F, Michel P, Gauducheau E, Lemeshow S, Salamon R, the EuroSCORE study group. Eur J Cardiothorac Surg. 1999;16:9-13.

6. Penicka M, Tousek P, De Bruyne B, Wijns W, Lang O, Madaric J, et al. Myocardial positive preejection velocity accurately detects presence of viable myocardium, predicts recovery of left ventricular function and bears a prognostic value after surgical revascularization. Eur Heart J. 2007;28:1366-73.

7. Kim RJ, Wu E, Rafael A, Chen EL, Parker MA, Simonetti O, et al. The use of contrast-enhanced magnetic resonance imaging to identify reversible myocardial dysfunction. N Engl J Med. 2000;343:1445-53.

8. Penicka M, Bartunek J, De Bruyne B, Vanderheyden M, Goethals M, De Zutter M, et al. Improvement of left ventricular function after cardiac resynchronization therapy is predicted by tissue Doppler imaging echocardiography. Circulation. 2004;209:978-83.

9. Bader H, Garrigue S, Lafitte S, Reuter S, Jais P, Haissaguerre M, et al. Intra-left ventricular electromechanical asynchrony. A new independent predictor of severe cardiac events in heart failure patients. J Am Coll Cardiol. 2004;43:248-56.

10. Cho GY, Song JK, Park WJ, Han SW, Choi SH, Doo YC, et al. Mechanical dyssynchrony assessed by tissue Doppler imaging is a powerful predictor of mortality in congestive heart failure with normal QRS duration. J Am Coll Cardiol. 2005; 46:2237-43

11. Yusuf S, Zucker D, Peduzzi P, Fisher LD, Takaro T, Kennedy JW, et al. Effect of coronary artery bypass graft surgery on survival: overview of 10-year results from randomised trials by the Coronary Artery Bypass Graft surgery Trialists Collaboration. Lancet. 1994;344:563-70.

12. Tarakji KG, Brunken R, McCarthy PM, Al-Chekakie MO, Abdel-Latif A Pothier CE, et al. Myocardial viability testing and the effect of early intervention in patients with advanced left ventricular systolic dysfunction. Circulation. 2006; 113:230-7.

13. Samady H, Elefteriades JA, Abbott BG, Mattera JA, McPherson CA, Wackers FJTh. Failure to improve left ventricular function after coronary revascularization for ischemic cardiomyopathy is not associated with worse outcome. Circulation. 1999;100:1298-304.

14. Cleland JG, Daubert JC, Erdmann E, Freemantle N, Gras D, Kappenberger L, et al. The effect of cardiac resynchronization on morbidity and mortality in hear failure. N Engl J Med. 2005;352:1539-49. 
15. Penicka M, Vanderheyden M, Geelen P, Mortier L, Goethals M, Verstreken S, et al. Tissue Doppler predicts long-term clinical outcome after cardiac-resynchronization therapy. Int J Cardiol. 2008;124:40-6.

16. Shapira OM, Hunter CT, Anter E, Bao Y, DeAndrade K, Lazar HL, et al. Coronary artery bypass grafting in patients with severe left ventricular dysfunctionearly and mid-term outcomes. J Card Surg. 2006;21:225-32.

17. Argenziano M, Spotnitz HM, Whang W, Bigger JT Jr, Parides M, Rose EA. Risk stratification for coronary bypass surgery in patients with left ventricular dysfunction: analysis of the coronary artery bypass grafting patch trial database. Circulation. 1999;100(19 suppl):II119-24.

18. Topkara VK, Cheema FH, Kesavaramanujam S, Mercando ML, Cheema AF, Namerow PB, et al. Coronary artery bypass grafting in patients with low ejection fraction. Circulation. 2005;112(9 suppl):I344-50.

19. Filsoufi F, Rahmanian PB, Castillo JG, Chikwe J, Kini AS, Adams DH. Results and predictors of early and late outcome of coronary artery bypass grafting in patients with severely depressed left ventricular function. Ann Thorac Surg. 2007;84:808-16.

20. Mickleborough LL, Carson S, Tamariz M, Ivanov J. Results of revascularization in patients with severe left ventricular dysfunction. J Thorac Cardiovasc Surg. 2000;119:550-7.
21. Ascione R, Narayan P, Rogers CA, Lim KH, Capoun R, Angelini GD. Early and midterm clinical outcome in patients with severe left ventricular dysfunction undergoing coronary artery surgery. Ann Thorac Surg. 2003;76:793-9.

22. Kaul TK, Agnihotri AK, Fields BL, Riggins LS, Wyatt DA, Jones CR. Coronary artery bypass grafting in patients with an ejection fraction of twenty percent or less. J Thorac Cardiovasc Surg. 1996;111:1001-12.

23. Anderson RP. First publications from the Society of Thoracic Surgeons National Database. Ann Thorac Surg. 1994;57:6-7.

24. Nashef SA, Roques F, Hammill BG, Peterson ED, Michel P, Grover FL, et al. EuroSCORE Project Group. Validation of European System for Cardiac Operative Risk Evaluation (EuroSCORE) in North American cardiac surgery. Eur $J$ Cardiothorac Surg. 2002;22:101-5.

25. Nilsson J, Algotsson L, Höglund P, Lührs C, Brandt J. Early mortality in coronary bypass surgery: the EuroSCORE versus The Society of Thoracic Surgeons risk algorithm. Ann Thorac Surg. 2004;77:1235-9.

26. Ad N, Barnett SD, Speir AM. The performance of the EuroSCORE and the Society of Thoracic Surgeons mortality risk score: the gender factor. Interact Cardiovasc Thorac Surg. 2007;6:192-5. 